\title{
Inferences about visual mechanisms from monocular depth effects'
}

FRED ATTMEAVE AND RICHARD K, OLSON

UNIVERSITY OF OREGON

Two depth "cues," radial patterning and relative length, were presented in sufficiently pure form to permit inferences about underlying data-processing operations. Over 180 o's, the former property yielded a strong depth impression, the latter a weaker one. Depth effects from dot patterns were dependent on perceptual grouping of dots into radial lines.

The properties of a monocular field that give rise to depth impressions have not typically been specified with precision. Ideally, the specification of a given "cue" would be formally equivalent to a description of the information-processing required to abstract the relevant property. This level of description is not unattainable, but it demands unusual attention to stimulus-control.

The perception of depth in a figure like la (after Gibson, 1950) might be attributed to any or all of a number of properties: (1) The transverse lines vary in slope, as radii of a circle. (2) The distance of each such line from its neighbors increases (linearly) from left to right. (3) The distances between verticals increase from left to right in accordance with a perspective rule. (Either the 1-2 gradient or the 3 gradient can produce a depth impression without the other; see Gibson, 1950.) (4) However, contour-density (average amount of line per unit area) increases concomitantly with both of the preceding gradients from right to left. (5) Likewise, object-density (number of trapezoids or of line segments per unit area) increases from right to left. (6) Object-size (area of trapezoids or length of line segments) varies inversely. The criterion for separating these variables is simply that each corresponds to a different set of possible operations on the proximal stimulus-data.

Stimuli

Displays were designed to test the effects of two potential depth cues-radial arrangement of lines (1) and relative length of linear elements (6)-with other, normally confounded cues controlled as strategically as possible.

Figures $1 \mathrm{~b}$ and $1 \mathrm{c}$ employ the same principle of control: verticals are so spaced that average contourdensity remains constant across the figure. Although the verticals do not follow a perspective gradient, their spacing might be expected to oppose the radial effect. Object-size (area of trapezoid) and object density are shallow non-monotonic functions of horizontal locus (inverted-U and $U$ respectively); i.e., trapezoids at either side are a little larger than intermediate ones. If effective in the present displays,

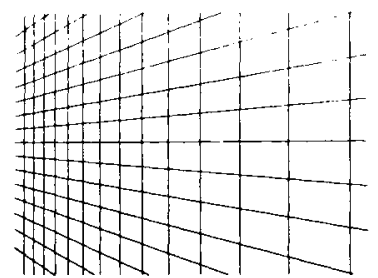

a. Demonstration

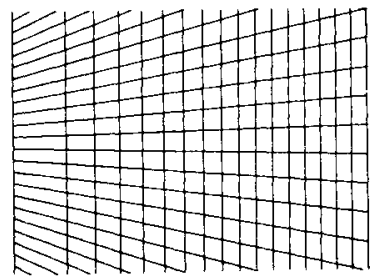

b. $18-0 \quad p<.001$

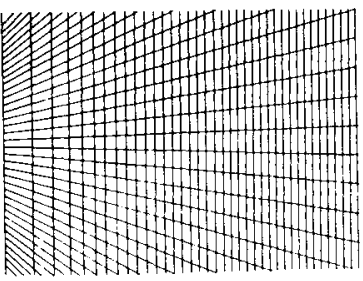

C. $17-1 \quad p<.001$

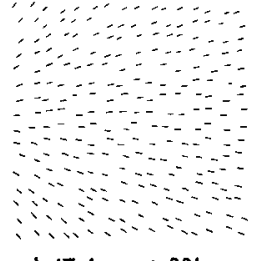

d. $17-1 \quad p<.001$

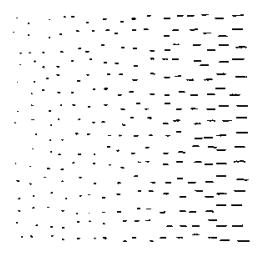

e. $17(6)^{-1(1)} \quad p<.001$
Fig. 1. Linear displays. Under each, number of O's reporting recession to the left and right respectively (in terms of the present orientation) is shown. Parenthetical numbers indicate forced choic es

these variables would create an impression of concavity, which does not in fact occur. Two aspects however.

This defect is remedied in Fig. 1d. Here, an appropriate radial segment of constant length was randomly positioned within each cell of a $16 \times 16$ construction grid, in such a manner as to break up alignments into precise rows and columns, without allowing elements to touch. It holds constant all the variables mentioned except slope-gradient.

In Figs. 1e and 1f, relative length of line segments is the independent variable. Object-density is held constant by randomly placing one element in each construction cell, Length gradients follow the perspective rules for physically equal elements, of the respective orientations shown, but elements in $1 \mathrm{c}$ are made horizontal rather than radial in deliberate violation of of radial lines-(1) and (2) above-remain confounded, 


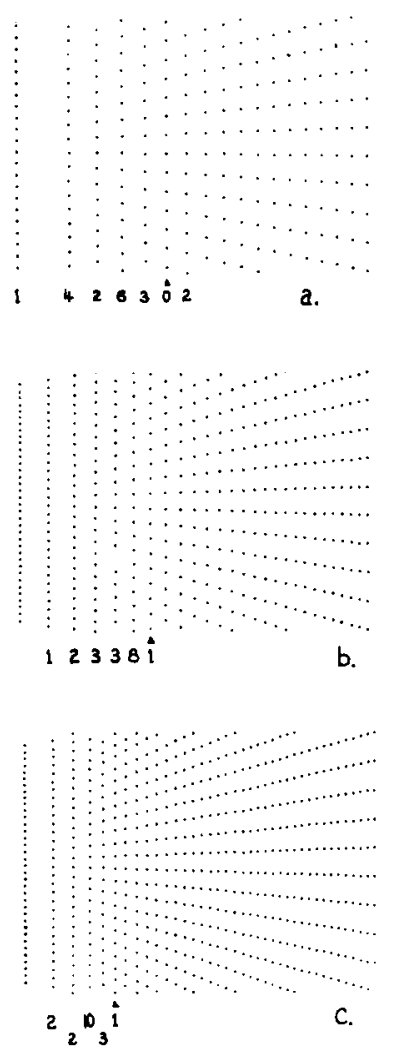

Fig. 2 Dot-patterns. The number under a column indicates the frequency with which the column was chosen as the inflection point of the surface. Pointer shows locus of equal spacing between columns and radii, measured at middle radius.

perspective rules. Two variables-contour density and space between elements - remain uncontrolled, but if effective, they will oppose the length variable.

Three dot patterns (Fig. 2) were used to study a relationship between perceptual grouping and depth perception. Dots are arranged in radial rows and vertical columns with dots closer in columns on one side and in radii on the other. The pointer indicates the locus of equidistance.

\section{Procedure}

Eighteen young adults, 5 men and 13 women, served as O's. The displays, in India ink on 9 in. $x 12$ in. cards, were presented at a distance of $5 \mathrm{ft}$. Figure la was shown as a demonstration. $O$ was then asked to tell, for each of the line displays (Figs. $1 c-f)$, the direction in which the surface seemed to recede. When necessary, a forced choice was demanded ("If you had to say that one side looks farther away ...") Order of presentation and left-right orientation of figures were randomly permuted for each $O$, except that each display was presented equally often in each orientation over all $O^{\prime} s$. The dot patterns were then presented, with order and orientation likewise varied. On the first one, $O$ was asked to report direction of recession and "anything else interesting" that he noticed. All but one $O$ (who had to be told what to look for) reported spontaneously that beyond some point the surface stopped receding, or flattened out. $O$ was then asked to indicate exactly where this discontinuity occurred in each display.

\section{Results and Discussion}

Results with $1 \mathrm{~b}$ and $1 \mathrm{c}$ show that a radial arrangement of lines effectively creates a depth-impression. Responses to $1 \mathrm{~d}$ show that a radial slope-gradient alone is effective, i. e., that distance between radial elements need not vary. Effects from $1 \mathrm{e}$ and $1 \mathrm{f}$ are weaker (possibly because of the opposing variables), but still show unequivocally the operation of a lengthgradient. (The imperfections of control mentioned earlier would present problems of interpretation only if the opposite result had been obtained.) In terms of information-processing, it seems minimally necessary to postulate (a) a slope-detecting operation, (b) a further mechanism responsive to functional relationships between slope and spatial position; likewise mechanisms for detection of (c) length and (d) lengthplace relationships.

The impression of a receding surface in the dot patterns (Fig. 2) clearly depends on perceptual grouping of dots into radial lines. The primary variable is relative proximity; however, the depth impression (and presumably the underlying grouping) typically perseverates for about two columns beyond the equidistance point. These results imply that proximitygrouping occurs prior to depth perception. A study by Rock \& Brosgole (1964) seems to show the reverse: they found grouping based on phenomenal rather than retinal proximity where the former was dependent upon (binocular) depth perception. It may be that the mechanisms for grouping and depth perception are not related in a fixed sequence, but that each operates on the output of the other in an iterative manner. Perseveration of perceived depth beyond the equidistance point would follow from this hypothesis. References

Gibson, J. J. The perception of the visual world. Cambridge: Riverside, 1950.

Rock, I.. \& Brosgole, L. Grouping based on phenomenal proximity. J. exp. Psychol., 1964, 67, 531-538.

\section{Note}

1. This research was supported by the Air Force Office of Scientific Research, Grant No. 9773-66. 